\title{
La importancia de la calidad en la universidad pública. La percepción del estudiante en la Universidad Autónoma del Carmen
}

Importance of quality in the public university. The perception of the student at the Autonomous University of Carmen

A importância da qualidade na universidade pública. Percepção do aluno na Universidad Autónoma del Carmen

David Martínez Luis

Universidad Autónoma del Carmen, Facultad de Ciencias Económicas Administrativas, México dmartinez@pampano.unacar.mx https://orcid.org/0000-0002-4747-9368

Alberto Pérez Fernández Universidad Autónoma del Carmen, Facultad de Ciencias Económicas Administrativas, México apfernandez@pampano.unacar.mx https://orcid.org/0000-0002-9397-4167

Lucio Alberto Pat Fernández Colegio de la Frontera Sur, México recurso90@hotmail.com https://orcid.org/0000-0003-1430-9343

José Félix García Rodríguez Universidad Juárez Autónoma de Tabasco, División Académica de Ciencias Económico Administrativas, México

jfgr55@hotmail.com https://orcid.org/0000-0002-7319-1472 


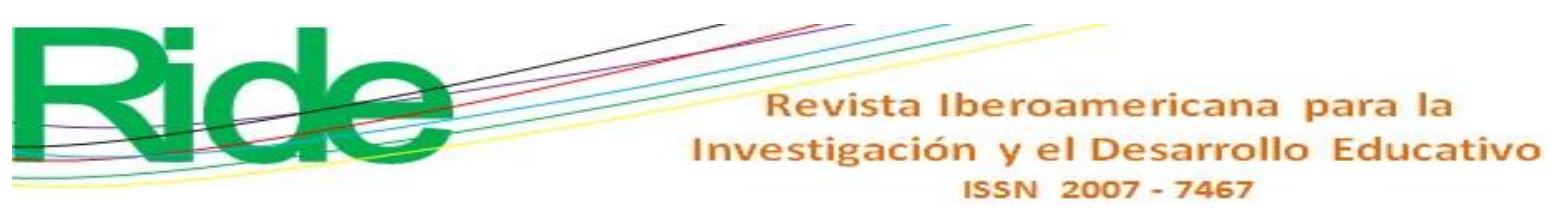

concluded that the university must focus its administrative policy on satisfying the needs of the student to reduce the decrease and improve terminal efficiency.

Keywords: education, expectation, income.

\section{Resumo}

As políticas públicas que liberalizaram a educação no México intensificaram a competição entre instituições de ensino. Nesse sentido, o ensino superior deve ser reconhecido como uma indústria de serviços, cujo objetivo é satisfazer as necessidades do cliente, principalmente em regiões com forte dinamismo econômico, como o município de Carmen, Campeche. O objetivo do artigo foi determinar a percepção que os alunos têm da qualidade de serviço da Universidade Autônoma do Carmen e os fatores que determinam essas percepções. Para determinar a qualidade do serviço, foi aplicado um questionário baseado no modelo SERVQUAL. Para analisar os fatores que afetam as percepções e expectativas dos alunos, vários modelos de regressão foram estimados usando o método dos mínimos quadrados ordinários. Os resultados mostram uma dificuldade da instituição em superar as expectativas dos alunos. Em uma cidade com forte dinamismo econômico e alta renda, o tempo de permanência dos estudantes e seu nível de renda determinam sua percepção da qualidade dos serviços oferecidos pela universidade. As percepções dos alunos podem melhorar à medida que os funcionários da faculdade prestam um serviço melhor. Conclui-se que a universidade deve focar sua política administrativa em satisfazer as necessidades do aluno para reduzir a evasão e melhorar a eficiência do terminal.

Palavras-chave: educação, avaliação, renda.

Fecha Recepción: Diciembre 2019

Fecha Aceptación: Mayo 2020

\section{Introducción}

El proceso de globalización y apertura económica ha elevado la competencia empresarial y la calidad de los bienes y servicios, los cuales determinan la permanencia de las empresas en el mercado (Rubinsztejn, Rivera-Torres y Grijalvo, 2015). En América Latina, se han realizado reformas con el objetivo de liberalizar la educación (Mollis, 2014) para aumentar la inversión privada y para crear más instituciones educativas. De acuerdo con Laval (2004), la privatización de la educación ha sido la consecuencia del deterioro de las condiciones materiales y pedagógicas de la escuela pública. 


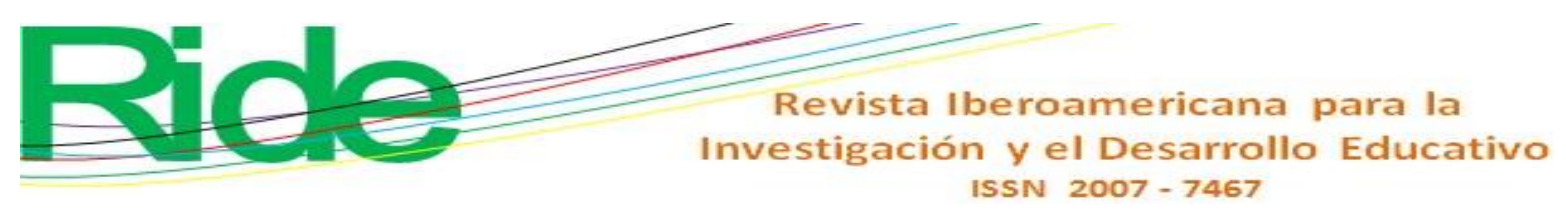

La liberalización de la educación ha intensificado la competencia entre universidades públicas y privadas, y ha servido de escenario para que los estudiantes elijan entre ellas tomando en cuenta el prestigio, las recomendaciones, la ubicación, los costos y la vanguardia en conocimientos. En México, por ejemplo, existen instituciones de educación privada que subsisten gracias al prestigio alcanzado, lo cual también ha servido para captar a una gran cantidad de alumnos a pesar del costo que ello implica (Fabela-Cárdenas y García-Treviño, 2014). El aumento de la competencia educativa ha obligado a las instituciones a trabajar para conseguir una mayor excelencia organizativa y ofrecer al alumno un servicio más satisfactorio desde una visión empresarial (Dos Santos, 2016).

La matrícula en educación superior en México pasó de menos de un millón de estudiantes en 1980 a 3.2 millones en 2014, crecimiento que fue impulsado no solo por el aumento de la matrícula en las instituciones de educación superior (IES) existentes, sino también por el surgimiento de nuevas universidades tanto públicas como privadas (Mendoza-Rojas, 2015). Aunado a lo anterior, existe una vinculación entre la asignación de fondos para las universidades y su desempeño institucional. Actualmente, los subsidios que se otorgan están vinculados al tamaño de la plantilla docente y matrícula (Moreno, 2017).

Las instituciones de educación deben considerar como política ofrecer servicios de calidad que se reflejen en la satisfacción de los usuarios para captar a más estudiantes y mejorar su eficiencia terminal (Juliá, Pérez y Meliá, 2014). La calidad es un factor que distingue a una universidad y le proporciona una ventaja frente a otras instituciones similares, lo cual determina su crecimiento en medio de una fuerte competencia (Naidu y Shuhada, 2016). Debido a esto, se puede afirmar que la educación superior debe ser reconocida como una industria de servicios, la cual se encarga de satisfacer las necesidades y expectativas de sus estudiantes o clientes (Boon, Ahmad, Ahmad y Ahmad, 2016). Por ese motivo, en este trabajo se aborda el tema de la calidad de la educación superior como un estudio de caso en la Universidad Autónoma del Carmen (UNACAR).

\section{Contexto regional y económico}

En Campeche se registró una matrícula en educación superior de 27914 alumnos para el ciclo 2016-2017. De la matrícula total en el estado, 8661 (31\%) estudiantes pertenecen al municipio del Carmen. La UNACAR es una institución pública ubicada en Ciudad del Carmen, Campeche, cuenta con ocho facultades y ofrece 33 programas de licenciatura cursados por 5827 


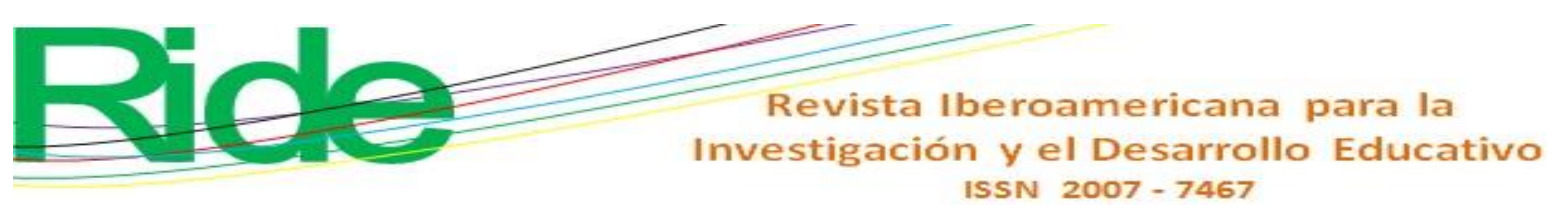

alumnos, lo que representa $67.3 \%$ de la matrícula del municipio y $20.9 \%$ de la matrícula estatal (Asociación Nacional de Universidades e Instituciones de Educación Superior [ANUIES], 2018).v

El municipio de Carmen se localiza en el sur del estado de Campeche y tiene una posición estratégica en la economía debido a su producción petrolera. La ciudad es la base principal de operaciones de las empresas relacionadas con la extracción de petróleo en la sonda de Campeche y es una importante fuente receptora de mano de obra de otras ciudades. En el año 2000, Carmen tenía una población de 172076 habitantes (Instituto Nacional de Estadística y Geografía [Inegi], 2000), cifra que aumentó a 221094 habitantes en 2015 (Inegi, 2015). El fuerte crecimiento de la población ha generado un aumento de la demanda de bienes y servicios, entre ellos los educativos.

Esta gran demanda de educación superior y el nivel de ingreso de la población fueron la base para la creación de diversas instituciones académicas, de ahí que actualmente existan ocho: tres públicas y cinco privadas. En algunas ciudades y regiones del país — como la Ciudad de México, Guadalajara, Monterrey - la demanda de educación superior es mayor a la oferta, y las universidades públicas tienen un fuerte índice de rechazo de aspirantes. Debido a estas circunstancias, una parte significativa de alumnos se ven en la necesidad de estudiar en universidades privadas. En Carmen la diferencia entre oferta y demanda es muy pequeña y la universidad pública está inmersa en un entorno de fuerte competencia. Aunado a lo anterior, el nivel de ingreso en Carmen permite que la población vea en la escuela pública y en la privada una opción válida, la cual suele ser determinada por la calidad de las instituciones.

En los últimos años, la ciudad sufrió una contracción de su actividad económica debido a la reforma petrolera y a la caída en los precios del petróleo. Esta crisis produjo una fuerte disminución del empleo, lo que obligó a muchos a emigrar a sus estados de origen, lo que afectó a los diversos sectores de la economía (Pérez, Rivas, Martínez y Venegas, 2018), entre ellos el educativo.

La competencia en este sector y la disminución de la demanda ha servido de estímulo para que la UNACAR diferencie sus servicios de los que prestan sus competidores, lo cual se ha efectuado tomando en cuenta los rápidos cambios suscitados en materia de oferta y demanda (Casadesús, Marimon y Alonso, 2010). Por ello, es elemental evaluar la calidad de los servicios que presta la UNACAR para mejorar la atención brindada al estudiante. 


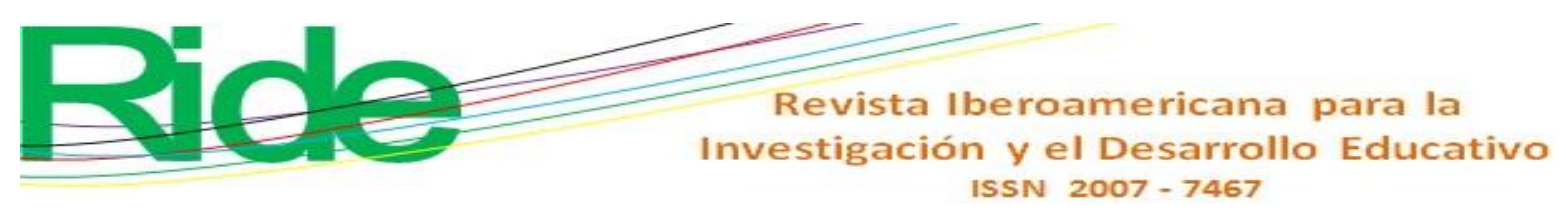

\section{Calidad del servicio en educación superior}

La calidad educativa se puede definir como la instrucción académica superior impartida en su conjunto (teórico-práctico) con el fin de permitir a los graduados responder a las exigencias de su profesión. Una institución que brinda una educación de calidad logra que sus egresados tengan aceptación en su entorno económico y en otras instituciones de educación superior (FabelaCárdenas y García-Treviño, 2014).

Para McCowan (2018) "la calidad en la educación superior puede referirse a cualquiera de las diversas funciones de la universidad" (p. 129). La excelencia en la educación debe incluir elementos relacionados con el proceso y el producto de la enseñanza, así como la organización y gestión de las instituciones universitarias (García-Jiménez, 2016).

Los servicios educativos primero se consumen y después se valoran de forma global (Jain, Sinha y Sahney, 2011) como una carrera universitaria. Rubinsztejn et al. (2015) conciben a la carrera universitaria como el conjunto de asignaturas organizadas en un periodo determinado, en un plan de estudios y coordinadas por docentes y administrativos. La calidad global de la carrera, por tanto, se refleja en la calidad del programa, las actividades realizadas en clases y las asignaciones complementarias (Sanchis, Gil-Saura y Berenguer-Contrí, 2015). Todo esto suele ser conocido por los aspirantes gracias a las opiniones de quienes han vivido la experiencia en cada institución, lo que en definitiva puede servir de sustento para tomar la decisión final de inscribirse en determinada universidad (Boyi, 2006). En pocas palabras, en la captación de alumnos, las referencias personales entre estudiantes es un factor muy importante (Marzo, Pedraja y Rivera, 2005).

Capelleras y Veciana (2001) mencionan que la decisión del estudiante para ingresar a la universidad está determinada por las competencias del profesorado, los planes de estudio, las instalaciones, los equipos y la organización. Otros estudios también analizan la calidad de la educación tomando en cuenta aspectos como instalaciones, innovación tecnológica, servicios de atención y relación entre profesor-alumno (Joseph y Joseph, 1997; Kwuan y Ng, 1999).

Oldfield y Baron (2000) afirman que la interacción entre profesores y alumnos es el elemento central del servicio educativo, lo que constituye un indicador importante de su calidad. El personal que presta el servicio es importante para quienes tienen ciertas expectativas de la organización. En este sentido, Zafiropoulos y Vrana (2008) evalúan la calidad en el servicio de las instituciones educativas a través de las posibles diferencias entre el personal y los estudiantes. 


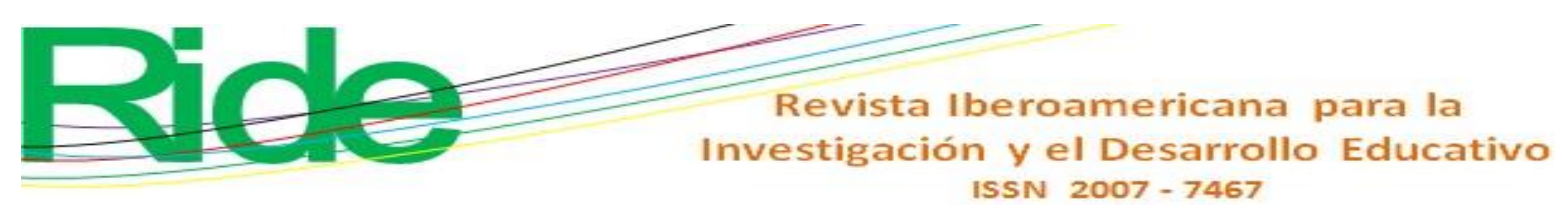

Metodología

La calidad en la educación se puede evaluar de manera externa tomando en cuenta la opinión de los egresados, los empleadores y los organismos acreditadores (Fabela-Cárdenas y García-Treviño, 2014) a través de instrumentos estandarizados (Vázquez, 2015). También se puede examinar de manera interna, considerando la opinión del estudiante sobre el papel del profesor, los jefes de departamento o directores, el personal administrativo y los rectores o vicerrectores (Durisová, Kucharcíková y Tokarcícová, 2015).

En esta investigación se ha realizado una evaluación interna de los servicios universitarios, por lo que la población objetivo fueron los estudiantes activos en la universidad. La UNACAR tiene una matrícula total de 5472 alumnos, cifra que sirvió de base para escoger una muestra de 359 estudiantes mediante la siguiente fórmula:

$$
n=\frac{Z^{2} p q N}{N e^{2}+Z^{2} p q}
$$

Determinada la muestra, se calculó el porcentaje de participación de cada facultad dentro de la matrícula total. A partir del porcentaje obtenido se calculó la muestra por facultad. Mediante un muestreo aleatorio simple se entrevistó a un total de 525 alumnos (tabla 1).

Tabla 1. Estructura de la muestra por facultad

\begin{tabular}{|l|c|c|c|c|}
\hline \multicolumn{1}{|c|}{ Facultad } & $\begin{array}{c}\text { Matrícula } \\
\text { (alumnos) }\end{array}$ & $\begin{array}{c}\text { Porcentaje } \\
(\%)\end{array}$ & $\begin{array}{c}\text { Muestra } \\
\text { calculada } \\
\text { por facultad }\end{array}$ & $\begin{array}{c}\text { Alumnos } \\
\text { entrevistados por } \\
\text { facultad }\end{array}$ \\
\hline $\begin{array}{l}\text { Ciencias de la } \\
\text { Información }\end{array}$ & 350 & 6 & 23 & 32 \\
\hline Ingeniería y Tecnología & 1130 & 21 & 74 & 82 \\
\hline Ciencias Naturales & 130 & 2 & 9 & 12 \\
\hline $\begin{array}{l}\text { Educación y } \\
\text { Humanidades }\end{array}$ & 379 & 7 & 25 & 62 \\
\hline $\begin{array}{l}\text { Ciencias Económicas } \\
\text { Administrativas }\end{array}$ & 950 & 17 & 62 & 147 \\
\hline Química & 743 & 14 & 49 & 60 \\
\hline Derecho & 550 & 10 & 36 & 43 \\
\hline Ciencias de la Salud & 1240 & 23 & 81 & 57 \\
\hline Total & 5472 & 100 & 359 & 525 \\
\hline
\end{tabular}

Fuente: Elaboración propia 




Revista Iberoamericana para la
Investigación y el Desarrollo Educativo
ISSN $2007-7467$

\section{Modelos determinantes de la percepción, expectativa y brecha}

La mayoría de los estudios empleados para determinar la calidad utilizan el modelo SERVQUAL, el cual puede ser usado para evaluar la expectativa y la percepción, aunque vale acotar que estas dos variables dependen de la edad, género, ingreso monetario y tiempo de permanencia en la universidad del estudiante (Rubinsztejn et al., 2015). Para evaluar estas relaciones se aplicó el siguiente modelo para cada una de las dimensiones del modelo SERVQUAL:

$$
Y_{m n}=\beta_{1} X_{1}+\beta_{2} X_{2}+\beta_{3} X_{3}+\beta_{4} X_{4}+e
$$

donde

$\mathrm{Y}_{\mathrm{mn}}=$ dimensión evaluada;

$\mathrm{X}_{1}=$ edad del estudiante;

$\mathrm{X}_{2}=$ género del estudiante;

$\mathrm{X}_{3}=$ tiempo que lleva como estudiante en la institución;

$\mathrm{X}_{4}=$ ingreso mensual familiar del estudiante;

$\beta_{\mathrm{i}}=$ parámetros a estudiar;

$\mathrm{m}=$ dimensión;

$n=$ aspecto a evaluar (expectativa, percepción o brecha), y

$e=$ término de error estocástico.

\section{Resultados}

\section{Confiabilidad del cuestionario y del análisis factorial}

El coeficiente alfa de Cronbach para expectativa, percepción y brecha fue cercano a 1, por lo que se puede afirmar que el cuestionario tiene alta confiabilidad. La medida de adecuación muestral Kaiser-Meyer-Olkin (KMO) obtenida para la expectativa, percepción y brecha fue mayor a 0.7, lo que indica que para los datos obtenidos es posible realizar el análisis factorial. La prueba de esfericidad de Bartlett obtenida para expectativa, percepción y brecha fue de 0.000. Con base en esta prueba, se puede asegurar que el análisis factorial es adecuado para explicar los datos generados por el cuestionario (tabla 2). 


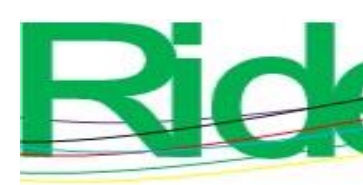

\section{Revista Iberoamericana para la Investigación y el Desarrollo Educativo \\ ISSN $2007-7467$}

Tabla 2. Medida Kaiser, Meyer-Olkin, prueba de esfericidad de Bartlett y prueba alfa Cronbach

\begin{tabular}{|c|c|c|c|c|}
\hline \multicolumn{2}{|l|}{ Prueba } & Expectativa & Percepción & Brecha \\
\hline \multicolumn{2}{|c|}{$\begin{array}{l}\text { Medida Kaiser-Meyer-Olkin de adecuación de } \\
\text { muestreo }\end{array}$} & .972 & .945 & .962 \\
\hline \multirow[t]{3}{*}{$\begin{array}{l}\text { Prueba de esfericidad de } \\
\text { Bartlett }\end{array}$} & $\begin{array}{l}\text { Aprox. Chi- } \\
\text { cuadrado }\end{array}$ & 12167.739 & 7464.582 & 8900.136 \\
\hline & Gl & 378 & 378 & 378 \\
\hline & Sig. & 0.000 & 0.000 & 0.000 \\
\hline Alfa de Cronbach & & 0.961 & 0.922 & 0.938 \\
\hline \multicolumn{2}{|c|}{ Alfa de Cronbach con elementos estandarizados } & 0.97 & 0.942 & 0.954 \\
\hline
\end{tabular}

Fuente. Elaboración propia

\section{Expectativa, percepción y brechas}

Los valores más altos que se obtuvieron en las expectativas de los estudiantes están relacionados con los profesores. La imagen del profesor, su capacidad y disponibilidad para atender al estudiante, así como su experiencia y capacidad para dar clases son aspectos que el alumno valora en sus expectativas. 
Tabla 3. Evaluación de los estudiantes sobre los servicios universitarios

\begin{tabular}{|c|c|c|c|c|c|c|}
\hline Ítem & \multicolumn{2}{|c|}{ Expectativa } & \multicolumn{2}{|c|}{ Percepción } & \multicolumn{2}{|c|}{ Brecha } \\
\hline & Media & $\begin{array}{l}\text { Desviació } \\
\text { n estándar }\end{array}$ & $\begin{array}{c}\text { Med } \\
\text { ia }\end{array}$ & $\begin{array}{l}\text { Desviac } \\
\text { ión } \\
\text { estándar }\end{array}$ & $\begin{array}{c}\text { Medi } \\
\mathrm{a}\end{array}$ & $\begin{array}{l}\text { Desviació } \\
\text { n estándar }\end{array}$ \\
\hline $\begin{array}{l}\text { T1. Las instalaciones y equipos } \\
\text { (edificios, talleres, laboratorios, } \\
\text { biblioteca, auditorio, zonas } \\
\text { verdes, baños) universitarios } \\
\text { están bien conservados. }\end{array}$ & 7.65 & 1.78 & 6.78 & 1.76 & -0.88 & 2.27 \\
\hline $\begin{array}{l}\text { T2. Las áreas públicas ron } \\
\text { agradables, } \\
\text { funcionales. }\end{array}$ & 7.98 & 3.93 & 7.16 & 1.68 & -0.83 & 4.03 \\
\hline $\begin{array}{l}\text { T3. Los profesores universitarios } \\
\text { se caracterizan por estar bien } \\
\text { vestidos. }\end{array}$ & 8.26 & 1.70 & 8.29 & 1.68 & 0.02 & 1.88 \\
\hline $\begin{array}{l}\text { T4. El personal administrativo se } \\
\text { caracteriza por tener un aspecto } \\
\text { limpio. }\end{array}$ & 8.52 & 1.54 & 8.45 & 1.57 & -0.09 & 1.76 \\
\hline $\begin{array}{l}\text { T5. Las instalaciones } \\
\text { universitarias están limpias. }\end{array}$ & 8.11 & 1.84 & 7.49 & 1.88 & -0.62 & 2.14 \\
\hline $\begin{array}{l}\text { T6. Las aulas tienen mobiliario y } \\
\text { equipo adecuados para dar y } \\
\text { recibir clases. }\end{array}$ & 8.07 & 1.80 & 7.41 & 4.85 & -0.67 & 5.01 \\
\hline $\begin{array}{l}\text { F1. Los servicios universitarios } \\
\text { (como servicios al estudiante, } \\
\text { servicios escolares, servicios } \\
\text { deportivos, bibliotecas, etc.) son } \\
\text { eficientes. }\end{array}$ & 7.93 & 1.91 & 7.00 & 2.01 & -0.95 & 2.39 \\
\hline $\begin{array}{l}\text { F2. El estudiante puede acceder } \\
\text { libremente a los servicios e } \\
\text { instalaciones universitarias. }\end{array}$ & 8.30 & 1.76 & 7.78 & 1.88 & -0.54 & 2.16 \\
\hline $\begin{array}{l}\text { F3. Se abarcan por completo } \\
\text { todos los temas de cada uno de los } \\
\text { cursos del programa educativo. }\end{array}$ & 8.32 & 1.70 & 7.93 & 1.65 & -0.40 & 1.99 \\
\hline $\begin{array}{l}\text { F4. Los documentos oficiales } \\
\text { expedidos por alguna instancia de } \\
\text { la universidad están libres de } \\
\text { errores. }\end{array}$ & 8.31 & 1.69 & 7.81 & 1.78 & -0.50 & 1.85 \\
\hline $\begin{array}{l}\text { F5. El personal administrativo } \\
\text { cumple con los horarios de } \\
\text { atención al estudiante, la entrega } \\
\text { de documentos, los horarios de } \\
\text { servicio según lo previamente } \\
\text { establecido. }\end{array}$ & 8.17 & 1.87 & 7.37 & 2.07 & -0.82 & 2.25 \\
\hline $\begin{array}{l}\text { C1. Los profesores siempre están } \\
\text { disponibles para asesorar al }\end{array}$ & 8.16 & 1.83 & 8.15 & 4.41 & -0.02 & 4.48 \\
\hline
\end{tabular}




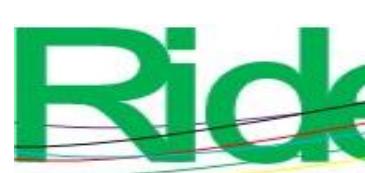

Revista Iberoamericana para la Investigación y el Desarrollo Educativo

ISSN 2007 - 7467

\begin{tabular}{|l|l|l|l|l|l|l|}
\hline alumno. & & & & & & \\
\hline $\begin{array}{l}\text { C2. Los servicios y trámites } \\
\text { universitarios se realizan con } \\
\text { rapidez. }\end{array}$ & 7.60 & 2.21 & 6.02 & 2.47 & -1.58 & 2.90 \\
\hline $\begin{array}{l}\text { C3. El personal directivo, } \\
\text { administrativo y de servicios está } \\
\text { siempre dispuesto a ayudar al } \\
\text { alumno. }\end{array}$ & 7.86 & 1.93 & 6.98 & 2.14 & -0.90 & 2.37 \\
\hline $\begin{array}{l}\text { C4. El personal de servicios al } \\
\text { estudiante (servicio social y } \\
\text { becas, movilidad, seguro } \\
\text { facultativo, tutoría, psicología, } \\
\text { etc.) está siempre disponible para } \\
\text { atender al alumno. }\end{array}$ & 7.87 & 2.07 & 6.85 & 2.28 & -1.04 & 2.57 \\
\hline $\begin{array}{l}\text { C5. El alumno tiene fácil acceso a } \\
\text { los directivos universitarios para } \\
\text { resolver sus problemas } \\
\text { académicos. }\end{array}$ & 7.75 & 2.03 & 6.80 & 2.23 & -0.95 & 2.46 \\
\hline
\end{tabular}

Fuente: Elaboración propia

Los alumnos de la UNACAR también tienen altas expectativas sobre los servicios e instalaciones, el cumplimiento de todos los programas de curso, los documentos expedidos por la universidad y la presentación del personal administrativo.

Respecto a la percepción, los estudiantes otorgaron calificaciones más altas a variables como la experiencia, capacidad y esfuerzo del profesor para dar clases (tablas 3 y 4). El aspecto con una menor calificación por parte del estudiante fue el sistema de inscripción. Un porcentaje importante de las evaluaciones bajas se relacionaron con la dimensión de empatía, lo que indica que en la universidad se requiere mejorar la atención al alumno por parte del personal administrativo, directivo y académico. Los alumnos consideraron que los profesores no se esfuerzan del todo en conocer y adaptarse a sus necesidades. Otro aspecto con baja calificación en la percepción fue el estado físico de las instalaciones.

Por otra parte, las brechas calculadas alcanzaron valores negativos, lo que quiere decir que las percepciones del alumno fueron menores a sus expectativas. La mayor insatisfacción se presentó en los servicios administrativos (p. ej., métodos de inscripción, selección de materias y atención al estudiante en trámites de servicio social, becas, movilidad, seguro facultativo, tutoría, psicología, entre otros). 
Tabla 4. Evaluación de los estudiantes sobre los servicios universitarios

\begin{tabular}{|c|c|c|c|c|c|c|}
\hline Ítem & \multicolumn{2}{|c|}{ Expectativa } & \multicolumn{2}{|c|}{ Percepción } & \multicolumn{2}{|c|}{ Brecha } \\
\hline & $\begin{array}{c}\text { Medi } \\
\text { a }\end{array}$ & $\begin{array}{l}\text { Desviac } \\
\text { ión } \\
\text { estándar }\end{array}$ & $\begin{array}{c}\text { Medi } \\
\mathrm{a}\end{array}$ & $\begin{array}{l}\text { Desviac } \\
\text { ión } \\
\text { estándar }\end{array}$ & $\begin{array}{c}\text { Medi } \\
\text { a }\end{array}$ & $\begin{array}{l}\text { Desviaci } \\
\text { ón } \\
\text { estándar }\end{array}$ \\
\hline $\begin{array}{l}\text { G1. Los directivos, profesores y } \\
\text { administrativos de la universidad generan } \\
\text { confianza. }\end{array}$ & 7.99 & 1.89 & 7.60 & 2.00 & -0.39 & 2.10 \\
\hline $\begin{array}{l}\text { G2. Los profesores tienen experiencia y } \\
\text { están capacitados en los cursos que } \\
\text { imparten. }\end{array}$ & 8.39 & 1.78 & 8.33 & 1.68 & -0.06 & 1.94 \\
\hline $\begin{array}{l}\text { G3. Los métodos de inscripción y selección } \\
\text { de materias son eficientes. }\end{array}$ & 7.86 & 2.23 & 5.32 & 2.93 & -2.54 & 3.40 \\
\hline $\begin{array}{l}\text { G4. Las opciones de titulación son } \\
\text { suficientes para garantizar la obtención del } \\
\text { título profesional. }\end{array}$ & 8.11 & 1.86 & 7.43 & 2.08 & -0.68 & 2.24 \\
\hline $\begin{array}{l}\text { G5. Las instalaciones universitarias } \\
\text { cumplen con las normas de seguridad. }\end{array}$ & 8.24 & 3.71 & 7.35 & 2.09 & -0.90 & 3.96 \\
\hline $\begin{array}{l}\text { E1. En clases, el profesor se esfuerza para } \\
\text { que los temas del curso queden claros a cada } \\
\text { alumno. }\end{array}$ & 8.33 & 1.76 & 8.16 & 1.78 & -0.18 & 2.00 \\
\hline $\begin{array}{l}\text { E2. Los directivos (rector, directores de } \\
\text { facultad, director de servicios al estudiante, } \\
\text { etc.) se esfuerzan por conocer las } \\
\text { necesidades del alumno. }\end{array}$ & 7.67 & 2.34 & 6.13 & 2.59 & -1.56 & 2.84 \\
\hline $\begin{array}{l}\text { E3. El profesor se esfuerza por conocer las } \\
\text { necesidades de cada alumno. }\end{array}$ & 7.72 & 2.09 & 6.98 & 2.21 & -0.73 & 2.38 \\
\hline $\begin{array}{l}\text { E4. En la universidad los alumnos son lo } \\
\text { más importante (el alumno es primero). }\end{array}$ & 7.70 & 2.26 & 6.65 & 2.41 & -1.07 & 2.75 \\
\hline $\begin{array}{l}\text { E5. Los horarios de los servicios } \\
\text { universitarios, incluidos los cursos, se } \\
\text { adaptan a las necesidades del alumno. }\end{array}$ & 7.80 & 2.15 & 6.75 & 3.60 & -1.07 & 3.70 \\
\hline $\begin{array}{l}\text { E6. El personal de servicios al estudiante } \\
\text { (becas, servicio social, movilidad, tutorías, } \\
\text { psicología, prácticas profesionales) se } \\
\text { esfuerza por resolver los problemas del } \\
\text { alumno. }\end{array}$ & 7.66 & 2.20 & 6.69 & 2.46 & -0.98 & 2.59 \\
\hline $\begin{array}{l}\text { E7. Los tutores y gestores de carrera se } \\
\text { preocupan por resolver los problemas del } \\
\text { alumno. }\end{array}$ & 7.99 & 2.16 & 7.56 & 2.40 & -0.45 & 2.47 \\
\hline
\end{tabular}

Fuente: Elaboración propia

Las brechas con valores negativos cercanos a cero surgieron en aspectos como la presentación o vestimenta del profesor, el profesor abarca todos los temas del programa de curso, genera confianza en el estudiante y se esfuerza para que los temas del curso sean claros para el 




Revista Iberoamericana para la Investigación y el Desarrollo Educativo ISSN 2007 - 7467

alumno. El estudio también refleja un buen papel de los tutores y gestores de carrera y su compromiso por resolver los problemas del alumno.

\section{Análisis factorial}

Tabla 5. Matriz de componentes rotados para expectativa, percepción y brechas

\begin{tabular}{|c|c|c|c|c|c|c|c|c|c|c|c|}
\hline \multirow[t]{2}{*}{ Ítem } & \multicolumn{2}{|c|}{$\begin{array}{l}\text { Componentes } \\
\text { expectativas }\end{array}$} & \multicolumn{5}{|c|}{ Componentes percepción } & \multicolumn{4}{|c|}{ Componentes brechas } \\
\hline & 1 & 2 & 1 & 2 & 3 & 4 & 5 & 1 & 2 & 3 & 4 \\
\hline $\mathrm{T} 1$ & & & & & & .630 & & & & .537 & \\
\hline $\mathrm{T} 2$ & & & & & & .646 & & & & .509 & \\
\hline $\mathrm{T} 3$ & & .699 & & & .706 & & & & & .587 & \\
\hline $\mathrm{T} 4$ & & .754 & & & .721 & & & & & .653 & \\
\hline T5 & & .754 & & & .557 & & & & & .677 & \\
\hline T6 & & .655 & & & & .652 & & & .522 & & \\
\hline F1 & & .703 & .612 & & & & & & .627 & & \\
\hline $\mathrm{F} 2$ & & .645 & .529 & & & & & & .504 & & \\
\hline $\mathrm{F} 3$ & & .695 & & & .636 & & & & & .652 & \\
\hline $\mathrm{F} 4$ & & .647 & & & .530 & & & & & & \\
\hline F5 & & .635 & .561 & & & & & & .528 & & \\
\hline $\mathrm{C} 1$ & & & & & & & .869 & & & & .839 \\
\hline $\mathrm{C} 2$ & & & .665 & & & & & & .564 & & \\
\hline $\mathrm{C} 3$ & 670 & & .678 & & & & & .557 & .527 & & \\
\hline $\mathrm{C} 4$ & .662 & & .781 & & & & & .501 & .583 & & \\
\hline $\mathrm{C5}$ & .662 & & .726 & & & & & & .560 & & \\
\hline G1 & .655 & & & & & & & & & & \\
\hline G2 & .639 & & & .519 & .597 & & & & & .637 & \\
\hline G3 & .696 & & .501 & & & & & .535 & & & \\
\hline G4 & .653 & & & .533 & & & & .527 & & & \\
\hline G5 & & & & .527 & & & & & .556 & & \\
\hline E1 & .722 & & & .565 & .510 & & & .633 & & .522 & \\
\hline E2 & .788 & & & .627 & & & & .753 & & & \\
\hline E3 & .789 & & & .719 & & & & .791 & & & \\
\hline $\mathrm{E} 4$ & .820 & & & .663 & & & & .748 & & & \\
\hline E5 & .824 & & & & & & & .606 & & & \\
\hline E6 & .825 & & .605 & & & & & .665 & & & \\
\hline E7 & .779 & & & .653 & & & & .657 & & & \\
\hline
\end{tabular}

Fuente: Elaboración propia

Método de extracción: Análisis de componentes principales.

Método de rotación: Varimax con normalización Kaiser. 


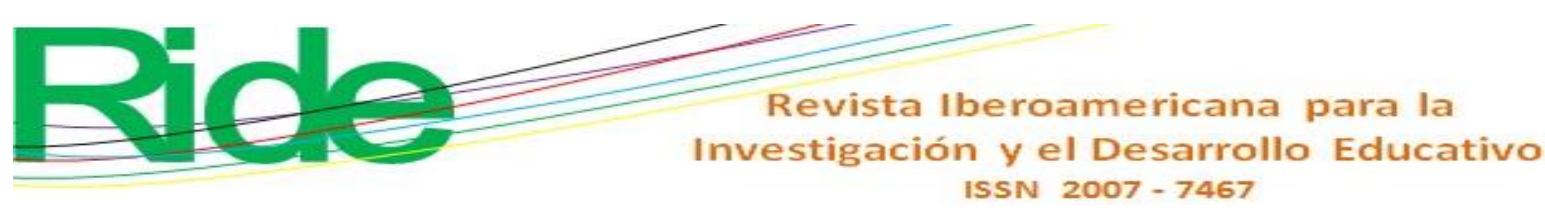

La tabla 6 describe las características de género, ingreso, tiempo de asistir a la universidad y la edad de los alumnos entrevistados. En los resultados estadísticos de los modelos de regresión se observa que la edad, género, tiempo de estancia en la universidad e ingreso familiar del estudiante determinan $95.6 \%$ de sus expectativas (promedio $\mathrm{R}^{2}=0.956$ ). En un análisis individual, los estimadores del ingreso y género no son confiables; por lo tanto, las expectativas del alumno están determinadas por la edad y el tiempo de permanencia en la universidad (tabla 7).

Asimismo, la edad, género, tiempo de estancia en la universidad e ingreso familiar determinan $95.6 \%$ de su percepción (promedio $\mathrm{R}^{2}=0.956$ ). Los estimadores para la edad, tiempo e ingreso son confiables a excepción del estimador del ingreso para la dimensión de tangibilidad. No se observa una relación entre la percepción y el género del estudiante debido a que los estimadores obtenidos no son confiables. Los estudiantes perciben una mejor calidad en los servicios de la universidad mientras más edad tienen. El tiempo que el alumno lleva en la universidad tiene un efecto negativo en su percepción de calidad de los servicios (tabla 7).

Las brechas se calculan por la diferencia entre percepción y expectativas y determinan si el alumno está satisfecho con el servicio. En promedio, los factores analizados determinan $18.3 \%$ de la satisfacción del alumno. Al analizar los coeficientes para las brechas se observa que el ingreso presenta estimadores confiables, con errores de $6 \%$ o menos; por lo tanto, la satisfacción del estudiante por los servicios de la UNACAR es determinada por su ingreso. Los niveles de error de los estimadores para la edad, tiempo de estancia en la universidad y género no indican que estas variables influyan sobre las brechas (tabla 7). 
Tabla 7. Coeficientes obtenidos para expectativa, percepción y brechas por cada dimensión

\begin{tabular}{|c|c|c|c|c|c|c|}
\hline Ecuación/dimensión & Edad & Género & Tiempo & Ingreso & $\mathbf{R}^{2}$ & $\operatorname{Pr}>\mathbf{F}$ \\
\hline \multicolumn{7}{|c|}{ Expectativa } \\
\hline \multirow[t]{2}{*}{ Tangibilidad } & 0.42017 & 0.0689 & -0.3783 & 0.0000043 & 0.956 & $<0.0001$ \\
\hline & $<0.0001$ & 0.7122 & $<0.0001$ & 0.6443 & & \\
\hline \multirow[t]{2}{*}{ Fiabilidad } & 0.4272 & 0.0569 & -0.3852 & 0.0000023 & 0.958 & $<0.0001$ \\
\hline & $<0.0001$ & 0.7612 & $<0.0001$ & 0.8000 & & \\
\hline \multirow[t]{2}{*}{ Capacidad de respuesta } & 0.4157 & 0.1152 & -0.4558 & $\begin{array}{c}- \\
0.0000014 \\
\end{array}$ & 0.9406 & $<0.0001$ \\
\hline & $<0.0001$ & 0.5887 & $<0.0001$ & 0.8893 & & \\
\hline \multirow[t]{2}{*}{ Garantía } & 0.4196 & 0.0129 & -0.3146 & $\begin{array}{c}- \\
0.0000077 \\
\end{array}$ & 0.941 & $<0.0001$ \\
\hline & $<0.0001$ & 0.9532 & 0.0003 & 0.4721 & & \\
\hline \multirow[t]{2}{*}{ Empatía } & 0.4175 & -0.0068 & -0.439 & 0.0000061 & 0.934 & $<0.0001$ \\
\hline & $<0.0001$ & 0.9763 & $<0.0001$ & 0.5777 & & \\
\hline \multirow[t]{2}{*}{ Promedio expectativa } & 0.42 & 0.04964 & -0.3946 & $\begin{array}{c}- \\
0.0000017 \\
\end{array}$ & 0.956 & $<0.0001$ \\
\hline & $<0.0001$ & 0.7897 & $<0.0001$ & 0.8486 & & \\
\hline \multicolumn{7}{|c|}{ Percepción } \\
\hline \multirow[t]{2}{*}{ Tangibilidad } & 0.4192 & -0.279 & -0.4172 & $\begin{array}{c}- \\
0.0000015 \\
\end{array}$ & 0.9466 & $<0.0001$ \\
\hline & $<0.0001$ & 0.1567 & $<0.0001$ & 0.1102 & & \\
\hline \multirow[t]{2}{*}{ Fiabilidad } & 0.4215 & 0.0295 & -0.5216 & -0.00001 & 0.9574 & $<0.0001$ \\
\hline & $<0.0001$ & 0.8656 & $<0.0001$ & 0.068 & & \\
\hline \multirow[t]{2}{*}{ Capacidad de respuesta } & 0.3969 & 0.1213 & -0.5668 & -0.00029 & 0.9352 & $<0.0001$ \\
\hline & $<0.0001$ & 0.5397 & $<0.0001$ & 0.0029 & & \\
\hline \multirow[t]{2}{*}{ Garantía } & 0.4016 & 0.0161 & -0.4427 & -0.000091 & 0.9463 & $<0.0001$ \\
\hline & $<0.0001$ & 0.9309 & $<0.0001$ & 0.0005 & & \\
\hline \multirow[t]{2}{*}{ Empatía } & 0.3916 & -0.0576 & -0.4887 & -0.000023 & 0.9221 & $<0.0001$ \\
\hline & $<0.0001$ & 0.7923 & $<0.0001$ & 0.0303 & & \\
\hline \multirow[t]{2}{*}{ Promedio percepción } & 0.4061 & -0.0339 & -0.4874 & -0.000023 & 0.9559 & $<0.0001$ \\
\hline & $<0.0001$ & 0.841 & $<0.0001$ & 0.0056 & & \\
\hline \multicolumn{7}{|c|}{ Brecha } \\
\hline \multirow[t]{2}{*}{ Tangibilidad } & -0.00099 & -0.3489 & -0.0388 & -0.00002 & 0.0744 & $<0.0001$ \\
\hline & 0.9319 & 0.1071 & 0.6436 & 0.0634 & & \\
\hline \multirow[t]{2}{*}{ Fiabilidad } & -0.0058 & -0.0273 & -0.1364 & -0.000018 & 0.1397 & $<0.0001$ \\
\hline & 0.55 & 0.8792 & 0.0518 & 0.0424 & & \\
\hline \multirow[t]{2}{*}{ Capacidad de respuesta } & -0.0188 & 0.0061 & -0.1111 & -0.000028 & 0.1846 & $<0.0001$ \\
\hline & 0.1158 & 0.9782 & 0.1971 & 0.0115 & & \\
\hline \multirow[t]{2}{*}{ Garantía } & -0.018 & 0.00324 & -0.1281 & -0.00024 & 0.175 & $<0.0001$ \\
\hline & 0.1377 & 0.9885 & 0.143 & 0.0279 & & \\
\hline Empatía & -0.0258 & -0.051 & -0.0496 & -0.000017 & 0.1516 & $<0.0001$ \\
\hline
\end{tabular}






Soe y Barua, 2013). Por lo anterior, el profesor debe diseñar e implementar estrategias de enseñanza y evaluación de manera constante (Yarmohammadian, Mozaffary y Esfahani, 2011). Las brechas negativas obtenidas en el estudio obligan a la UNACAR a centrar sus esfuerzos en optimizar la formación pedagógica y disciplinar de los profesores.

Las brechas negativas más altas del estudio se registraron en los servicios que la universidad presta al estudiante (ajenos a la actividad docente), es decir, los departamentos de tutorías, becas, intercambio académico, servicio social, seguro facultativo, selección y sistema de alta de cursos, entre otros. La mala calidad de estos servicios son un problema porque en ocasiones los alumnos consideran más importante los aspectos relacionados con el servicio que los vinculados con la docencia (Hill, 1995). La universidad, por tanto, debe trabajar en mejorar la atención al alumno y centrar su atención en la eficiencia de su personal administrativo para mejorar la calidad de sus servicios (Giannini, 2015). Mejorar la administración de los recursos humanos y materiales y optimizar la cultura pedagógica de la universidad pueden elevar la calidad y la matrícula (MacCowan, 2018; Rodríguez-Ponce, Pedraja-Rejas, Delgado-Almonte y Ganga-Contreras, 2017).

Rodríguez, Ariza y Ramos (2014) encontraron un débil poder explicativo del nivel socioeconómico sobre el rendimiento académico. A pesar de las diferencias que pudieran existir en el nivel de ingreso de los estudiantes, la forma en que los estudiantes construyen sus percepciones sobre la calidad de las instituciones es similar en una universidad tanto pública como privada (Alvarado, Morales y Aguayo, 2016).

Las expectativas de los estudiantes están determinadas por la información con que cuentan, sus experiencias previas y estudios anteriores. Estas expectativas varían con el tiempo y cambian de acuerdo con la vivencia del estudiante dentro de la institución (Rubinsztejn et al., 2015). Li y Kaye (1999) también encontraron que las percepciones de la calidad del servicio tienden a disminuir durante el periodo de consumo del servicio. Los resultados encontrados también indican una relación inversa entre el ingreso familiar y la percepción del estudiante. Esto supone que a mayor ingreso las exigencias por el servicio se elevan. Rubinsztejn et al., (2015) explican que la percepción del alumno sobre la calidad de servicios que recibe está determinada por la experiencia adquirida al avanzar en su carrera.

En el presente estudio se realizó un análisis de áreas muy generales dentro de la universidad. Dentro de estas áreas puede existir mucha diferencia en la calidad de servicio de los departamentos que la integran, aunque en la investigación no se pudieron detectar. Aun así, la metodología 


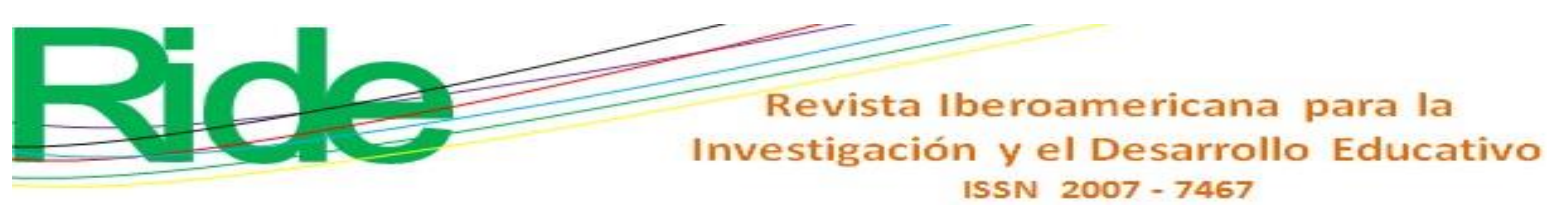

empleada permitirá hacer adaptaciones al estudio de departamentos específicos y contribuir a la implementación de políticas específicas en las instituciones educativas.

Las expectativas y percepciones del estudiante en general muestran la calidad de los servicios que se ofrecen. El mayor aporte de la investigación tiene que ver con la incorporación de factores que determinan estas percepciones y su cuantificación. En México se han creado universidades en diversas regiones del país, las cuales enfrentan diferentes exigencias. Al estudiar los factores que determinan las percepciones del consumidor, las universidades pueden detectar las necesidades de los alumnos y generar políticas internas que lleven a incrementar su matrícula y eficiencia terminal.

\section{Conclusiones}

Los estudiantes perciben que la calidad de los servicios de la UNACAR es menor a lo que esperan al ingresar a la institución. Los servicios relacionados con las funciones docentes son mejor evaluados, mientras que los servicios relacionados con los sistemas de inscripción y servicios al estudiante reciben calificaciones bajas, lo cual refleja la poca atención que reciben los estudiantes por parte del personal que labora en estas áreas.

Las expectativas de los estudiantes de la UNACAR están determinadas por sus edades y por sus experiencias dentro de la universidad, mientras que sus percepciones están vinculadas con sus edades, vivencias dentro de la institución y niveles de ingreso. Al obtener la diferencia entre expectativa y percepción se observa que el factor determinante en la calidad de los servicios universitarios está determinado por el nivel de ingreso. Los alumnos de mayor edad tienen una más alta exigencia por la calidad de los servicios universitarios, y mientras más tiempo permanecen dentro de la institución tienden a percibir una menor calidad en los servicios. El dinamismo económico de la ciudad, producto de la actividad petrolera, es un factor importante en la evaluación del estudiante sobre la universidad. Las instituciones educativas inmersas en un entorno económico similar deben de centrar su política en el mejoramiento continuo de la calidad de los servicios ofrecidos al estudiante.

En síntesis, la universidad debe enfocarse en capacitar constantemente a sus profesores y al personal que se relaciona de forma directa con el estudiante. En un entorno de fuerte competencia con instituciones privadas, el personal y los servicios que facilitan el proceso de formación del estudiante son fundamentales para lograr su satisfacción. 


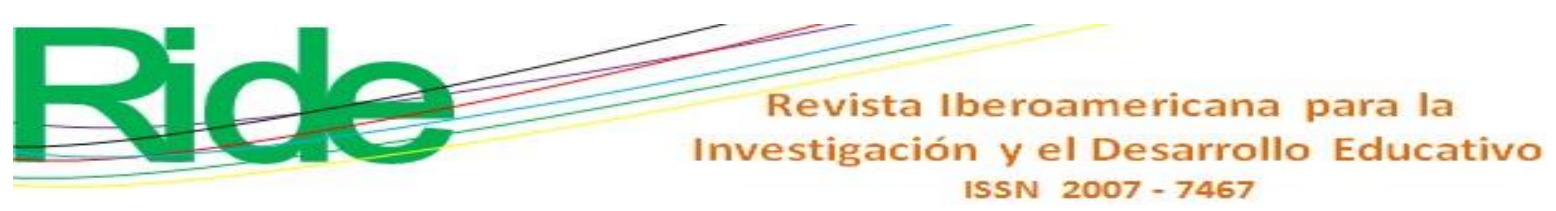

García-Jiménez, E. (2016). Concepto de excelencia en enseñanza superior universitaria. Educación Médica, 17(3), 83-87. Doi: 10.1016/j.edumed.2016.06.003

Gathoni, N. and van der Walt, T. (2019). Evaluating library service quality at the Aga Khan University library: Application of a total quality management approach. Journal of Librarianship and Information Science, 51(1), 123-136. Doi: 10.1177/0961000616679725

Giannini, M. (2015). Organization and quality in school education. Procedia, Social and Behavioral Sciences, 174(1), 1735-1739. Doi:10.1016/j.sbspro.2015.01.831

Gregory, J. L. (2019). Applying SERVQUAL: Using service quality perceptions to improve student satisfaction and program image. Journal of Applied Research in Higher Education, ahead-of-print No. ahead-of-print. Doi: 10.1108/JARHE-12-2018-0268

Hill, M. (1995). Managing service quality in higher education: the role of the student as primary consumer. Quality Assurance in Education, 3(3), 10-21. Doi: $10.1108 / 09684889510093497$

Hortigüela, D., Ausín, V., Delgado, V. y Abella. V. (2017). Análisis de la importancia de los criterios de evaluación y el reconocimiento académico docente universitario como indicadores de calidad educativa en España. Revista de la Educación Superior, 46(181), 75-87. Doi: 10.1016/j.resu.2016.10.002

Instituto Nacional de Estadística y Geografía [Inegi] (2000). Censo Nacional de Población y Vivienda.

Instituto Nacional de Estadística y Geografía [Inegi] (2015). Encuesta intercensal.

Jain, R., Sinha, G. and Sahney, S. (2011). Conceptualizing service quality in higher education. Asian Journal on Quality, 12(3), 296-314. Doi:10.1108/15982681111187128

Jerez, Ó., Orsini, C. y Hasbún, B. (2016). Atributos de una docencia de calidad en la educación superior: una revisión sistemática. Estudios Pedagógicos, 42(3), 483-506. Doi: 10.4067/S0718-07052016000400026

Joseph, M. and Joseph, B. (1997). Service quality in education: A student perspective. Quality in Assurance in Education, 5(1), 15-21. Doi:10.1108/0968488971015654

Juliá, J. F., Pérez, J. A. y Meliá, E. (2014). El cambio necesario de la universidad española, ante un nuevo escenario económico. Interciencia, 39(1), 60-67. Recuperado de http://hdl.handle.net/10251/65526 


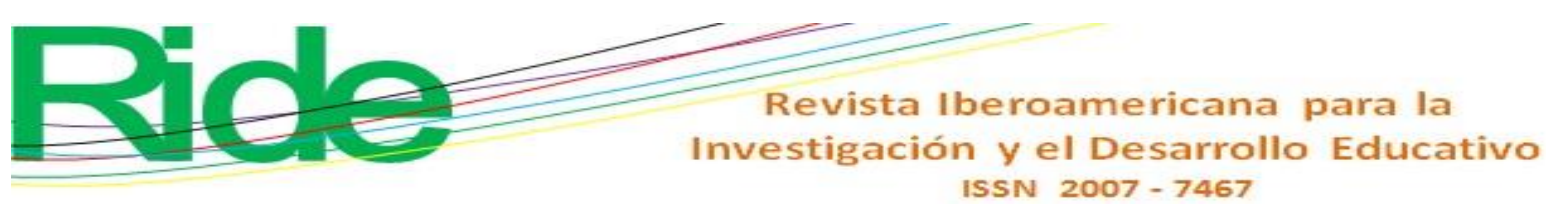

Kwuan, P. and Ng, P. (1999). Quality indicators in higher education-comparing Hong Kong and China's students. Managerial Auditing Journal, 14(1), 20-27. Doi: $10.1108 / 02686909910245964$

Laval, C. (2004). La escuela no es una empresa. Barcelona: Paidós.

Li, R. Y. and Kaye, M. (1999). Measuring service quality in the context of teaching: a study on the longitudinal nature of students' expectations and perceptions. Innovations Education and Teaching International, 36(2), 145-154.

Makoe, M. and Nsamba, A. (2019). The gap between student perceptions and expectations of quality support services at the University of South Africa. American Journal of Distance Education, 33(2), 132-141. Doi: 10.1080/08923647.2019.1583028

Marzo, M., Pedraja, M. and Rivera, P. (2005). A new management element for universities: satisfaction with the offered courses. International Journal of Educational Management, 19(6), 505-526. Doi: 10.1108/09513540510617454

Matsumoto, R. (2014). Desarrollo del modelo SERVQUAL para la medición de la calidad del servicio en la empresa de publicidad Ayuda Experto. Perspectivas, (34), 181-209. Recuperado de http://www.redalyc.org/articulo.oa?id=425941264005

McCowan, T. (2018). Quality of higher education in Kenya: Addressing the conundrum. International Journal of Education Development, 60(1), 128-137. Doi: 10.1016/j.ijedudev.2017.11.002

Mejías, A. A. (2005). Modelo para medir la calidad del servicio en los estudios universitarios de postgrado. Universidad, Ciencia y Tecnología, 9(34), 81-85. Recuperado de http://ve.scielo.org/scielo.php?script=sci_arttext\&pid=S1316-48212005000200004

Mendoza-Rojas, J. (2015). Ampliación de la oferta de educación superior en México y creación de instituciones públicas en el periodo 2001-2012. Revista Iberoamericana de Educación Superior, 16(6), 3-32. Doi:10.22201/iisue.20072872e.2015.16

Mollis, M. (2014). Administrar la crisis de la educación pública y evaluar la calidad universitaria en América Latina: dos caras de la misma reforma educativa. Revista de la Educación Superior, 43(169), 25-45. Recuperado de http://www.redalyc.org/articulo.oa?id=60430753003

Moreno, C. I. (2017). Las reformas en educación superior pública en México: rupturas y continuidades. Revista de la Educación Superior, 46(182), 27-44. Doi: 10.1016/j.resu.2017.03.001 


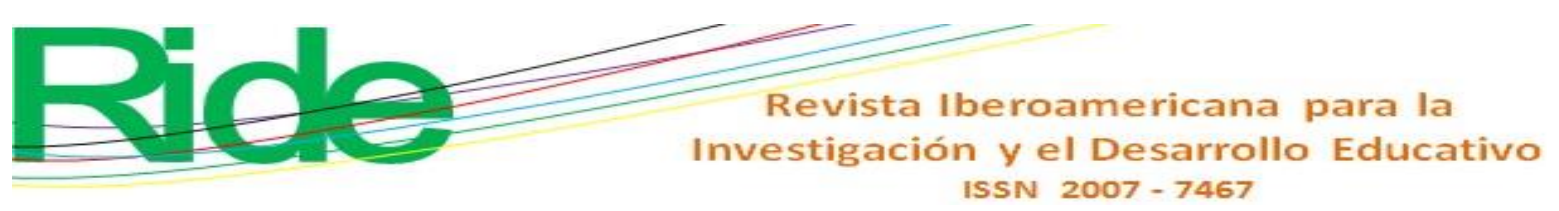

Naidu, P. and Shuhada, N. E. (2016). A comparative study on quality of education received by students of private universities versus public universities. Procedia, Economics and Finance, 35(1), 659-666. Doi:10.1016/s2212-5671(16)00081-2

Oldfield, B. M. and Baron, S. (2000). Students' perception of service quality in a UK university business and management faculty. Quality Assurance in Education, 8(2), 85-95. Doi: 10.1108/09684880010325600

Parasuraman, A., Zeithaml, V. A. and Berry, L. L. (1994). Alternative scales for measuring service quality: a comparative assessment based on psychometric diagnostic criteria. Journal of Retailing, 70(3), 201-230. Doi:10.1016/0022-4359(94)90033-7

Pérez, F. A., Rivas, M. I., Martínez, D. y Venegas, J. A. (2018). La restructuración de PEMEX y su efecto en los salarios y el mercado de trabajo en Ciudad del Carmen, Campeche. Análisis Económico, 33(82), 111-124. Recuperado de http://www.redalyc.org/articulo.oa?id=41355807007

Prugsamatz, S., Heaney, J. G. and Alpert, F. (2007). Measuring and investigating pretrial multiexpectations of service quality within the higher education context. Journal of Marketing for Higher Education, 17(1), 17-47. Doi: 10.1300/J050v17n01_04

Rodríguez, G. Ariza, M. y Ramos, J. L. (2014). Calidad institucional y rendimiento académico. El caso de las universidades del Caribe colombiano. Perfiles Educativos, 36(143), 10-29. Recuperado de https://www.iisue.unam.mx/perfiles/numeros/2014/143

Rodríguez-Ponce, E., Pedraja-Rejas, L., Delgado-Almonte, M. y Ganga-Contreras, F. (2017). La relación entre la gestión financiera y la calidad en las instituciones de educación superior. Interciencia, 42(2), 119-126. Recuperado de http://www.redalyc.org/articulo.oa?id=33949912008

Rubinsztejn, G., Rivera-Torres, P. y Grijalvo, M. (2015). Calidad y recomendación en educación superior: el rol de la experiencia del estudiante. Interciencia, 40(12), 816-826.

Rushton, A., Croucher, P., and Baker, P. (2017). The handbook of logistics and distribution manage- ment: Understanding the supply chain (6 ${ }^{\text {th }}$ ed.). London, UK: Kogan Page.

Sanchis, M., Gil-Saura, I. y Berenguer-Contrí, G. (2015). Dimensionalidad del servicio universitario: una aproximación desde un enfoque de marketing. Revista Iberoamericana de Educación Superior, 6(15), 26-49. Doi:10.1016/S20072872(15)30002-0 




Singh, S., Pai, D. R., Sinha, N. K., Kaur, A., Soe, H. H. K. and Barua, A. (2013). Qualities of an effective teacher: what do medical teachers think? BMC Medical Education, 13(128), 1-7. Recuperado de http://www.biomedcentral.com/1472-6920/13/128

Stefano, N. M., Casarotto, N., Barichelo, R. and Sohn, A. P. (2015). A fuzzy SERVQUAL based method for evaluated of service quality in the hotel industry. Procedia CIRP, 30, 433 438.

Vázquez, M. G. (2015). La calidad de la educación. Reformas educativas y control social en América Latina. Revista de Estudios Latinoamericanos, (60), 93-124. Doi: 10.1016/j.larev.2014.10.001

Vera, J. (2013). Atributos de la calidad del servicio de la telefonía móvil para clientes mexicanos y su impacto en la satisfacción y en la lealtad hacia la marca. Contaduría y Administración, 58(3), 39-63. Doi:10.1016/S0186-1042(13)71221-X

Vergara, J. C. y Quesada, V. M. (2011). Análisis de la calidad en el servicio y satisfacción de los estudiantes de Ciencias Económicas de la Universidad de Cartagena mediante un modelo de ecuaciones estructurales. Revista Electrónica de Investigación Educativa, 13(1), 108-122.

Yarmohammadian, M. H., Mozaffary, M. and Esfahani, S. S. (2011). Evaluation of quality of education in higher education based on Academic Quality Improvement Program (AQIP) Model. Procedia, Social and Behavioral Sciences, 15(1), 2917-2922. Doi: 10.1016/j.sbspro.2011.04.214

Zafiropoulos, C. and Vrana, V. (2008). Service quality assessment in a Greek higher education institute. Journal of Business Economics and Management, 9(1), 33-45. Doi: 10.3846/1611-1699.2008.9.33-45

Zeithaml, V., Berry, L. and Parasuraman, A. (1993). The nature and determinants of customer expectations of service. Journal of the Academy of Marketing Science, 21(1), 1-12. Doi: 10.1177/0092070393211001 


\begin{tabular}{|l|l|}
\hline Rol de Contribución & Autor (es) \\
\hline Conceptualización & David Martínez y Alberto Pérez (igual) \\
\hline Metodología & David Martínez, Alberto Pérez, Lucio Pat (igual) \\
\hline Software & David Martínez, José Félix García (igual) \\
\hline Validación & David Martínez, José Félix García, Lucio Pat (igual) \\
\hline Análisis Formal & $\begin{array}{l}\text { David Martínez, Alberto Pérez, Lucio Pat, José Félix García } \\
\text { (igual) }\end{array}$ \\
\hline Investigación & $\begin{array}{l}\text { David Martínez (principal). Apoyo: Alberto Pérez, Martha E. } \\
\text { Córdova, Vanesa MarinaLópez, Pamela Martínez, Karen } \\
\text { Samantha Dionicio, Nolbeth Tagano. }\end{array}$ \\
\hline Recursos & Universidad Autónoma del CArmen \\
\hline Curación de datos & David Martínez \\
\hline $\begin{array}{l}\text { Escritura - Preparación del } \\
\text { borrador original }\end{array}$ & $\begin{array}{l}\text { David Martínez (principal). Apoyo: Alberto Pérez Fernández, } \\
\text { Lucio Pat, José Félix García }\end{array}$ \\
\hline $\begin{array}{l}\text { Escritura } \\
\text { edición }\end{array}$ & $\begin{array}{l}\text { David Martínez, Alberto Pérez Fernández, Lucio Pat, José Félix } \\
\text { García }\end{array}$ \\
\hline Visualización & David Martínez \\
\hline Supervisión & David Martínez, Alberto Pérez \\
\hline Adquisición de fondos & David Martínez \\
\hline
\end{tabular}

